\title{
Numerical Computations for Flow Patterns and Force Statistics of Three Rectangular Cylinders
}

\author{
Hamid Rahman, ${ }^{1}$ Shams-ul-Islam ${ }^{D},{ }^{2}$ Waqas Sarwar Abbasi, ${ }^{3}$ Raheela Manzoor, ${ }^{4}$ \\ Fazle Amin, ${ }^{1}$ and Zeeshan Alam (iD) \\ ${ }^{1}$ Department of Mathematics \& Statistics, Bacha Khan University, Charsadda 24420, Pakistan \\ ${ }^{2}$ Mathematics Department, COMSATS University, Islamabad 44000, Pakistan \\ ${ }^{3}$ Mathematics Department, Air University, Islamabad 44000, Pakistan \\ ${ }^{4}$ Mathematics Department, SBK Women's University, Quetta 87300, Pakistan \\ ${ }^{5}$ College of Mathematics \& Statistics, Baise University, Baise 533000, China \\ Correspondence should be addressed to Zeeshan Alam; zeeshan_alam84@hotmail.com
}

Received 23 March 2021; Revised 25 July 2021; Accepted 11 November 2021; Published 29 November 2021

Academic Editor: Chiara Bedon

Copyright (c) 2021 Hamid Rahman et al. This is an open access article distributed under the Creative Commons Attribution License, which permits unrestricted use, distribution, and reproduction in any medium, provided the original work is properly cited.

\begin{abstract}
In this work, numerical simulations are performed in order to study the effects of aspect ratio (AR) and Reynolds number (Re) on flow characteristics of three side-by-side rectangular cylinders for fixed spacing ratio $(g)$, using the lattice Boltzmann method (LBM). The Reynolds number varies within the range $60 \leq \operatorname{Re} \leq 180$, aspect ratio is between 0.25 and 4 , and spacing ratio is fixed at $g=1.5$. The flow structure mechanism behind the cylinders is analyzed in terms of vorticity contour visualization, time-trace analysis of drag and lift coefficients, power spectrum analysis of lift coefficient and variations of mean drag coefficient, and Strouhal number. For different combinations of AR and Re, the flow is characterized into regular, irregular, and symmetric vortex shedding. In regular and symmetric vortex shedding the drag and lift coefficients vary smoothly while reverse trend occurs in irregular vortex shedding. At small AR, each cylinder experiences higher magnitude drag force as compared to intermediate and large aspect ratios. The vortex shedding frequency was found to be smaller at smaller AR and increased with increment in AR.
\end{abstract}

\section{Introduction}

Fluid flow around multiple bluff bodies like circular, square, or rectangular cylinders has practical significance in many branches of science and engineering such as aerodynamical systems, high-rise buildings, underwater structures, overhead power-line bundles, microelectromechanical systems, and cooling towers. In some of the cases, these structures have circular or square cross section, but mostly the structures are of rectangular cross section. The rectangular cross section components can also be found in beams, fences, and other building construction materials. Based on these applications, the study of flow around rectangular cross section cylinders needs much attention compared to circular/square cylinders because in available literature, very limited studies have been conducted regarding the flow around such cross-sectional structures. The main objective of the present study is to fully understand the flow characteristics of three side-by-side rectangular cylinders in terms of vorticity contours, time-trace analysis of drag and lift coefficients (CD and CL), power spectrum analysis of lift coefficient, fluid forces, and its resulting effects. Flow structure mechanism behind multiple rectangular cylinders depends on many nondimensional parameters, which are Reynolds number (Re), spacing ratio $(g)$, and aspect ratio (AR). Another aim of this study is to understand the effect of these nondimensional parameters on flow around three side-by-side rectangular cylinders.

In open literature, numerous studies can be found for flow around an isolated single cylinder. Islam et al. [1] numerically found single primary vortex frequency for all Re with the periodic nature of physical parameters. According to Golani and Dhiman [2] the drag coefficient decreases while shedding frequency increases by increasing Re. Gera 
et al. [3] found significant variations in Strouhal number (ST) under the effect of Reynolds numbers. Numerical simulations were conducted by Islam et al. [4] to study the effect of AR on flow around a single rectangular cylinder. They noticed a decrease in drag coefficient and ST with increment in $\operatorname{Re}$ for $\operatorname{Re}=100$ and 150, while irregular variations in ST were found for $\mathrm{Re}=200$ and 250. Abdollah et al. [5] reported longer near wake region for rectangular cylinder. Okajima [6] concluded that at high Reynolds numbers the value of Strouhal changes abruptly when the value of AR is about 2.6 or 6. From this discussion, it is deduced that the Reynolds number and aspect ratio are important flow parameters which affect the flow structure mechanism behind an isolated single cylinder.

Contrary to single cylinder flow, flow around two sideby-side cylinders is more complex because of the gap between the cylinders from which a complex flow structure arises. Agrawal et al. [7] concluded that the vortices develop at $\operatorname{Re}=10$ and the flow becomes oscillating at $\mathrm{Re}=30$ behind the two cylinders at $g=2.5$. Furthermore, they observed that the amalgamation of the vortices was gradual in case of synchronized flow pattern and in case of flip-flopping flow pattern the amalgamation was rapid. Kang [8] numerically examined the flow behind two side-by-side circular cylinders and observed steady flow, single bluff-body, deflected flow, flip-flopping, in-phase synchronized, antiphase synchronized flow patterns. His analysis showed that both Re and $g$ affect the wake structure mechanism, but the effect of $g$ was much stronger than Re in case of small $g$. Alam and Zhou [9] investigated the flow structure mechanism around two sideby-side square cylinders and categorized the flow into the single bluff-body, asymmetric wake, transition, and couple street regimes. Han et al. [10] numerically observed the single bluff-body, the gap flow interface, and the antiphase synchronized flow patterns for flow behind two side-by-side square cylinders. Zhang et al. [11] reported that vortex shedding changed from one pattern to another with Re for two side-by-side circular cylinders.

The flow structure mechanism around three or more cylinders is much complex because of the multiple gaps between the cylinders. Some studies for flow around multiple circular as well as square cylinders, arranged side-by-side, can also be found in the open literature. But the effect of aspect ratio and Reynolds numbers is not studied for flow behind side-by-side rectangular cylinders, to the best of authors' knowledge. Sewatkar et al. [12] numerically investigated the combined effect of Reynolds number and spacing ratio for flow around a row of cylinders and found that Re affects the flow structure mechanism behind the square cylinders at $g=3$ and 4 . They also found that the secondary cylinder interaction frequencies diminished at large Re. Harichandan and Roy [13] found increment in ST for flow around multiple cylinders for increasing Re. Han et al. [14] categorized the flow around three side-by-side circular cylinders into eight different patterns depending on $\mathrm{Re}$ and g: single bluff-body, deflected flow, flip-flopping, nonidentical steady state flow, identical steady state flow, antiphase synchronized flow, in-phase synchronized, and combined in-phase and antiphase synchronized. They reported that $\mathrm{CD}$ and CL showed periodic oscillations for single bluffbody flow, static value for deflected pattern. For flipflopping pattern the drag and lift curve shows variation, for antiphase synchronized pattern the CL1 and CL2 show antiphase periodic behavior while CD1 and CD2 coincide, and for in-phase synchronized pattern the curve of CD2 and CD3 and CL1 and CL2 are in-phase. Kang [15] numerically analyzed the flow around three side-by-side circular cylinders and characterized the flow into modulation-synchronized, in-phase synchronized, flip-flopping, deflected, and single bluff-body flow patterns. Rahman et al. [16] found that, in case of multiple cylinders, as the spacing ratio increases the effect of gap flow vanishes and each cylinder behaves like a single isolated cylinder. They also observed the bistable, asymmetric, inphase-antiphase, and modulated synchronized flow patterns. Lattice Boltzmann simulations were carried out by Ul Islam et al. [17] in order to study the effect of equal and unequal spacing ratios on flow behind three side-by-side rectangular cylinders. They categorized the flow into bistable, asymmetric, in-phase-antiphase, and modulated synchronized flow patterns. They also reported that slight change in unequal spacing ratio caused drastic changes in fluid forces. Rahman et al. [18] characterized the flow behind three side-by-side rectangular cylinders into deflected, bistable, flip-flopping, chaotic, symmetric and synchronized flow patterns. Steady, modulated, symmetric, and periodic flow patterns were reported by Rahman et al. [19] for flow behind three side-by-side rectangular cylinders for different aspect ratios and Reynolds numbers. They found the aspect ratio more influential on flow characteristics of the cylinders compared to $\operatorname{Re}$ at large $g$ while for small spacing the fluid forces and flow structure mechanism changed abruptly due the jet flow generation within the gap.

From above literature review, it is clear that the analysis of flow around rectangular cross section cylinders was lacking and needs attention as compared to square or circular cylinders. It can also be deduced from the above discussions that $\mathrm{Re}, \mathrm{AR}$, and $g$ affect the flow characteristics around side-by-side arranged cylinders. Some reference data are available for the effect of Re and spacing ratio for flow past circular or square cylinders in side-by-side arrangement, but there is one reference paper [19] available regarding the effect of Re for flow behind three side-by-side rectangular cylinders. The first aim of the present study is to analyze the effect of aspect ratio and Reynolds number on flow structure mechanism around the three side-by-side cylinders at $g=1.5$ as it is a well observed fact that the flow becomes fully developed [14-16] at this spacing value. The second aim of the present study is to investigate the variation of flow 
induced forces and their dependence on $g$ and AR. The analysis of the effect of jet flow on fluid flow characteristics of three side-by-side rectangular cylinders is the third objective of the present study. The fourth objective of the present study is to enrich the database of fluid structure interaction problems.

\section{Numerical Details}

The lattice Boltzmann method (LBM) is a powerful technique for the computational modelling of a wide variety of complex fluid flow problems. LBM can be easily implemented to the problems having complex geometries and can easily handle the nonlinear term in Navier-Stokes equation (Sukop and Thorne [20]). LBM is second order accurate in both time and space and explicit in nature (Wolf-Gladrow [21]). Also the pressure term is calculated using the equation of state and there is no need to solve Poisson equation in each time-step (Mohamad [22]). Another advantage of LBM is that it can easily be parallelized due to its local nature (Guo et al. [23]). Based on these advantages, for the present study LBM is adopted as a numerical method. In this section a brief introduction of LBM will be discussed and for more details the readers are referred to [20-23].

The governing Navier-Stokes equations for the problem under consideration are

$$
\begin{gathered}
\nabla \cdot u=0, \\
\frac{\partial u}{\partial t}+(u \cdot \nabla) u=-\frac{1}{\rho} \nabla p+\nu \nabla^{2} u .
\end{gathered}
$$

Equation (1) represents the continuity while equation (2) represents the momentum equation. These equations can be recovered by applying the Chapman Enskog expansion (Chapman and Cowling [24]) to the discretized lattice Boltzmann equation given as

$$
\underbrace{g_{i}\left(x+e_{i} \delta t, t+\delta t\right)-g_{i}(x, t)}_{\text {Streaming }}=\underbrace{-1 / \tau\left(g_{i}(x, t)-g_{i}^{\mathrm{eq}}(x, t)\right)}_{\text {Collision }},
$$

where $g_{i}$ is the density evolution function at position $x$ and time $t$, and $\tau$ is the single relaxation time parameter which should be greater than 0.5 for stable simulations. In equation (3) the left-hand side represents the streaming step in which the particles propagate to the neighbor node while the righthand side of equation (3) represents the collision step in which the particles collide. The equilibrium distribution function $g_{i}^{\mathrm{eq}}$ is given by the relation

$$
g_{i}^{\mathrm{eq}}=\rho \omega_{i}\left[1+3\left(e_{i} \cdot u\right)-\frac{3}{2} u^{2}+\frac{9}{4}\left(e_{i} \cdot u\right)^{2}\right],
$$

where $\omega_{i}$ are the weighting coefficients. These weighting coefficients are different for different models used in LBM. For the two-dimensional nine-velocity-particles $\left(D_{2} Q_{9}\right)$ model, used in the present study, the weighting coefficients are

$$
\omega_{i}= \begin{cases}\frac{4}{9}, & i=0, \\ \frac{1}{9}, & i=1,2,3,4, \\ \frac{1}{36}, & i=5,6,7,8 .\end{cases}
$$

The mass density $(\rho)$ and the velocity $(u)$ are calculated as

$$
\begin{aligned}
& \rho=\sum_{i=0}^{8} g_{i}, \\
& u=\frac{1}{\rho} \sum_{i=1}^{8} e_{i} g_{i} .
\end{aligned}
$$

The speed of sound and pressure are calculated as

$$
\begin{aligned}
c_{s} & =\frac{e}{\sqrt{3}}, \\
p & =\rho c_{s}^{2} .
\end{aligned}
$$

The kinematic viscosity is calculated as

$$
\nu=\left(\tau-\frac{1}{2}\right) c_{s}^{2} \delta t
$$

\section{Statement of the Problem and Flow Parameters}

The schematic diagram of flow behind three side-by-side rectangular cylinders is presented in Figure 1. Three rectangular cylinders with height " $h$ " and width " $w$ " are exposed to uniform incoming flow with velocity $\left(u=U_{\max } ; v=0\right)$ in a channel of length " $L$ " and width " $W$." The cylinders are placed in the channel from bottom to top in side-by-side arrangement. Computational domain varies in stream-wise direction $(x)$ by varying the aspect ratios and fixed in transverse direction $(y)$ as $g=1.5$. In Figure $1, L_{1}$ represents the upstream length and $L_{2}$ represents the downstream length. Surface-to-surface distance between the cylinders is denoted by " $s$ " and $c_{1}, c_{2}$, and $c_{3}$ represent the lower, middle, and upper cylinder, respectively. On the lower and the upper wall of the computational domain and on the surfaces of each cylinder no-slip boundary condition is applied (Gallivan et al. [25]). At the outlet of the channel convective boundary condition is used for the smooth passage of flow (Sohankar et al. [26]). The flow parameters used in the current study are aspect ratio, spacing ratio, Reynolds number, Strouhal number, drag coefficient, lift coefficient, and mean drag coefficient and these parameters are defined as $\mathrm{AR}=w / h, g=s / h, \mathrm{Re}=U_{\max } h / v, \mathrm{ST}=f_{s} h / U_{\max }$ (where $f_{s}$ is the vortex shedding frequency), $\mathrm{CD}=2 F_{x} / \rho U_{\max }^{2} h$ (where $F_{x}$ is the force component along the $x$-axis), $\mathrm{CL}=$ $2 F_{y} / \rho U_{\max }^{2} h$ (where $F_{y}$ is the force component along $y$-axis), 


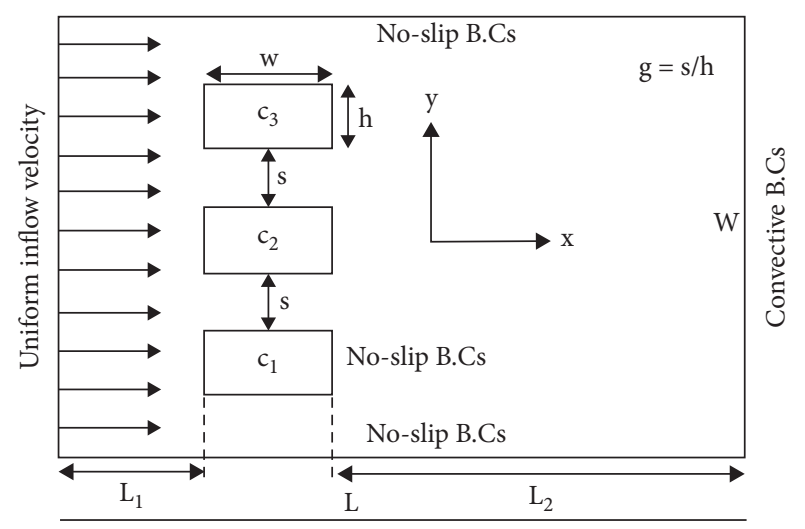

FIGURE 1: Schematic configuration of three side-by-side rectangular cylinders.

and $\mathrm{CD}$ mean $=\sum_{i=1}^{n} \mathrm{CD}(i) / n$ (where $n$ is the number of time-steps), respectively.

\section{Domain Independence and Code Validation}

For channel flow the selection of a suitable computational domain is essential. For this purpose, at three different upstream positions ( $L_{1}=6 \mathrm{~h}, 8 \mathrm{~h}$, and $10 \mathrm{~h}$ ) and downstream positions $\left(L_{2}=25 \mathrm{~h}, 30 \mathrm{~h}\right.$, and $\left.35 \mathrm{~h}\right)$ and three different widths of the channel ( $W=8 \mathrm{~h}, 9 \mathrm{~h}$, and $10 \mathrm{~h}$ ), the Strouhal number is computed in order to choose a suitable domain. The simulations are performed for three side-by-side rectangular cylinders at aspect ratio of 1.5 and a Reynolds number of 150 . The simulated results are tabulated in Table 1. Table 1 shows that the differences in results in the domain size $L_{1}=8 \mathrm{~h}, L_{2}=30 \mathrm{~h}, W=9 \mathrm{~h}$ and other domain size combinations are very small for all three cylinders. That is why in the current study, all the simulations are performed at $L_{1}=8 \mathrm{~h}, L_{2}=30 \mathrm{~h}$, and $W=9 \mathrm{~h}$ (excluding the cylinders and spacing ratios).

Before performing the computations of the current flow problem, first we have validated our code. For this purpose a code based on LBM is written for flow behind a single isolated square cylinder and after validation, this code is extended to flow around three side-by-side rectangular cylinders. Domain size selected for numerical investigations is $37 \mathrm{~h}$ in longitudinal direction, while $10 \mathrm{~h}$ is selected for transverse direction. To validate our code, computations for Strouhal number are done at $\mathrm{Re}=100$ and 150 and are compared with different experimental and numerical studies already available in the literature. This comparison is given in Table 2. From the table it is clear that, at $R e=100$, our results are in better agreement with the experimental measurements of Okajima [27] and Norberg [28] and numerical results of Sohankar et al. [29] and Gera et al. [3]. Also at $\mathrm{Re}=150$, our results are very close to the experimental work of Norberg [28] and numerical work of Sohankar et al. [29]. Some negligible discrepancies can be found which are due to the use of different domain size, numerical method, boundary conditions, etc. (Table 2). From the discussion above, it can be deduced that our results and the results of other researchers coincide for flow behind a single isolated
TABle 1: Domain independence study.

\begin{tabular}{lccc}
\hline \multicolumn{4}{c}{$g=1.5 ; \mathrm{AR}=1.5 ; \mathrm{Re}=150$} \\
\hline Cases & ST1 & ST2 & ST3 \\
$L_{1}=6 \mathrm{~h} ; L_{2}=30 \mathrm{~h} ; W=9 \mathrm{~h}$ & 0.1884 & 0.2241 & 0.1759 \\
$L_{1}=10 \mathrm{~h} ; L_{2}=30 \mathrm{~h} ; W=9 \mathrm{~h}$ & 0.1815 & 0.2310 & 0.1759 \\
$L_{1}=8 \mathrm{~h} ; L_{2}=25 \mathrm{~h} ; W=9 \mathrm{~h}$ & 0.1815 & 0.2241 & 0.1897 \\
$L_{1}=8 \mathrm{~h} ; L_{2}=35 \mathrm{~h} ; W=9 \mathrm{~h}$ & 0.1747 & 0.2241 & 0.1828 \\
$L_{1}=8 \mathrm{~h} ; L_{2}=30 \mathrm{~h} ; W=8 \mathrm{~h}$ & 0.2089 & 0.2103 & 0.1897 \\
$L_{1}=8 \mathrm{~h} ; L_{2}=30 \mathrm{~h} ; W=10 \mathrm{~h}$ & 0.1678 & 0.2310 & 0.1697 \\
$L_{1}=8 \mathrm{~h} ; L_{2}=30 \mathrm{~h} ; W=9 \mathrm{~h}$ & 0.2031 & 0.2311 & 0.2031 \\
\hline
\end{tabular}

TABLE 2: Validation of code for flow around a square cylinder.

\begin{tabular}{lccc}
\hline \multirow{2}{*}{ References } & \multicolumn{2}{c}{ Strouhal number } \\
& & $\mathrm{Re}=100$ & $\mathrm{Re}=150$ \\
\hline Present & Okajima [27] & $\begin{array}{c}0.14410 \\
(2.422 \%)\end{array}$ & $0.1420(8.3 \%)$ \\
Experimental & Norberg [28] & $0.1402(2.98 \%)$ & $\begin{array}{c}0.1500 \\
(2.84 \%)\end{array}$ \\
& $\begin{array}{c}\text { Sohankar et al. } \\
\text { [29] }\end{array}$ & $0.1450(0.48 \%)$ & $0.1610(4.3 \%)$ \\
Numerical & Gera et al. [3] & $0.1290(10.7 \%)$ & $0.1410(8.6 \%)$ \\
\hline
\end{tabular}

square cylinder. So, the current code can also be used for flow behind multiple cylinders' case.

\section{Results and Discussion}

In the present study, flow behind three side-by-side rectangular cylinders is analyzed for different Reynolds numbers, aspect ratios, and fixed spacing ratio $(g=1.5)$. The reason for fixing $g$ at 1.5 is that at $g=1.5$ the flow around side-by-side cylinders fully developed [14-16] and our main focus is to analyze the effect of Re and AR on flow structure mechanism and fluid forces. The Reynolds number is taken in the range from 60 to 180 . Values of Re selected for analysis are $\mathrm{Re}=60,80,100,120,140,160$, and 180 and aspect ratio is varied in the range from 0.25 to 4 . Selected values of different aspect ratios for analysis are $\mathrm{AR}=0.25,0.5,0.75,1,1.25,1.5$, $1.75,2,2.5,3,3.5$, and 4 . Important findings of this study are discussed below.

5.1. Effect of Reynolds Number on Flow Structure at Different Aspect Ratios. The analysis of the effect of Re on flow structure mechanism for all values of AR considered in this study revealed that the flow behavior for $0.25 \leq \mathrm{AR} \leq 1$, $1.25 \leq \mathrm{AR} \leq 2.5$, and $3 \leq \mathrm{AR} \leq 4$ is similar. So, for the sake of brevity only some representative cases of AR will be discussed here, which are $\mathrm{AR}=0.5,2$, and 4 .

The vorticity contour visualization for $\mathrm{Re}=60-180$ and $\mathrm{AR}=0.5$ is shown in Figure 2. The flow structure observed at $(\mathrm{AR}, \mathrm{Re})=(0.5,60)$ is termed as symmetric vortex shedding, which is further subdivided into nearly symmetric and symmetric flow patterns. Figure 2(a) shows nearly symmetric flow pattern, in which the vortices are generated only from lower and upper cylinder. These vortices diminish quickly in the down wake region, while the middle cylinder shows nearly steady 




(a)

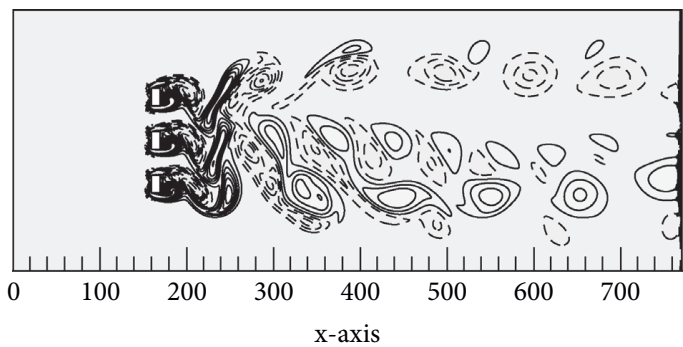

(c)

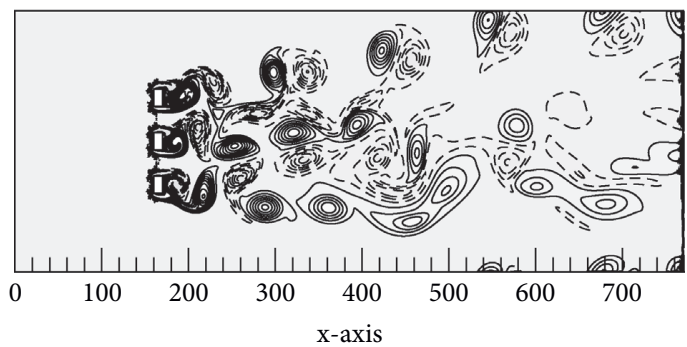

(e)



(b)

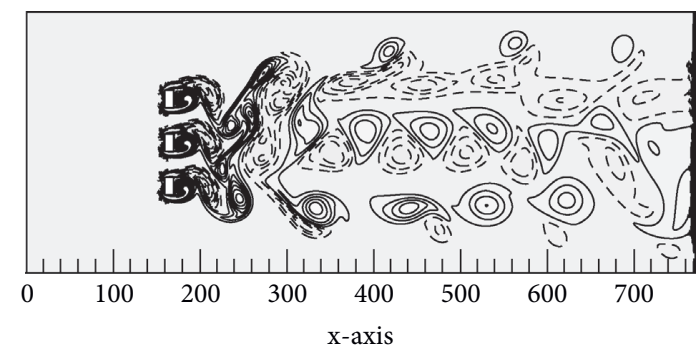

(d)

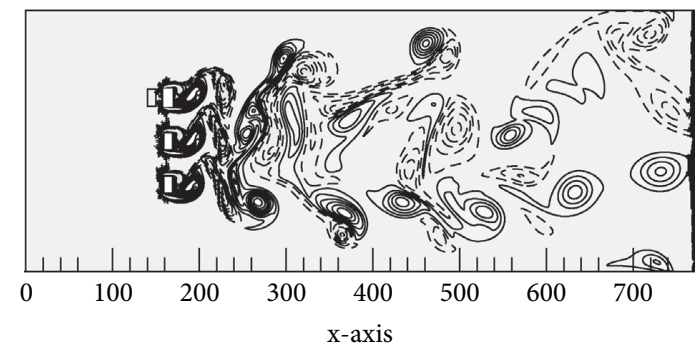

(f)

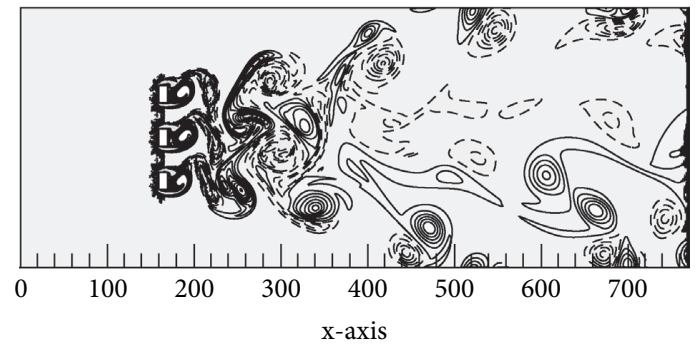

(g)

Figure 2: Vorticity contour visualizations at $A R=0.5$ and $\operatorname{Re}=60-180$. (a) $(A R, R e)=(0.5,60),(b)(A R, R e)=(0.5,80),(c)(A R, R e)=(0.5$, $100)$, (d) $(A R, R e)=(0.5,120),(e)(A R, R e)=(0.5,140)$, (f) $(A R, R e)=(0.5,160)$, and $(g)(A R, R e)=(0.5,180)$.

behavior. The time signals of lift coefficients for cylinder $c_{2}$ show nearly steady behavior while those for cylinders $c_{1}$ and $c_{3}$ are antiphase to each other (Figure 3(b)). The drag signals for nearly symmetric flow pattern are periodic with varying amplitude (see Figure 3(a)). It can be seen from Figure 3(a) that the lower and upper cylinder's drag are identical and both the cylinders $\left(c_{1}\right.$ and $c_{2}$ ) experience higher drag compared to middle cylinder $\left(c_{2}\right)$. Similarly, the lift coefficient magnitude of lower and upper cylinders is also identical but due to symmetry CL of these cylinders are mirror images of each other (Figure 3(b)). Inoue and Suzuki [30] also observed similar flow pattern for three side-by-side square cylinders. The vorticity contours shown in Figures 2(b)-2(g) represents the regular vortex shedding in which the generated vortices are either in-phase or antiphase or combined in-and-antiphase. The vortices shed from the upper and lower side of each cylinder merge with each other in down wake region. In-phase vortex shedding can be seen for (AR, $\operatorname{Re})=(0.5,100)$ and $(0.5,120)$ (see Figures $2(\mathrm{c})$ and $2(\mathrm{~d}))$. The shear layers detaching from cylinders $c_{1}-c_{2}$ and $c_{1}-c_{3}$ are antiphase with each other (see Figure 2(e)). The in-phase and antiphase vortex shedding behavior can also be verified from the lift signals shown in Figures 3(d) and 3(f). Figure 3(d) shows the in-phase vortex shedding while Figure 3(f) illustrates the antiphase vortex shedding. From Figure 3(h), it can be seen that the lift signals are antiphase for some time and then become inphase. That is why this pattern is termed as combined in-andantiphase flow pattern. The same flow pattern also occurs for $(A R, R e)=(0.5,80)$. The periodicity of lift signals for in-phase, 

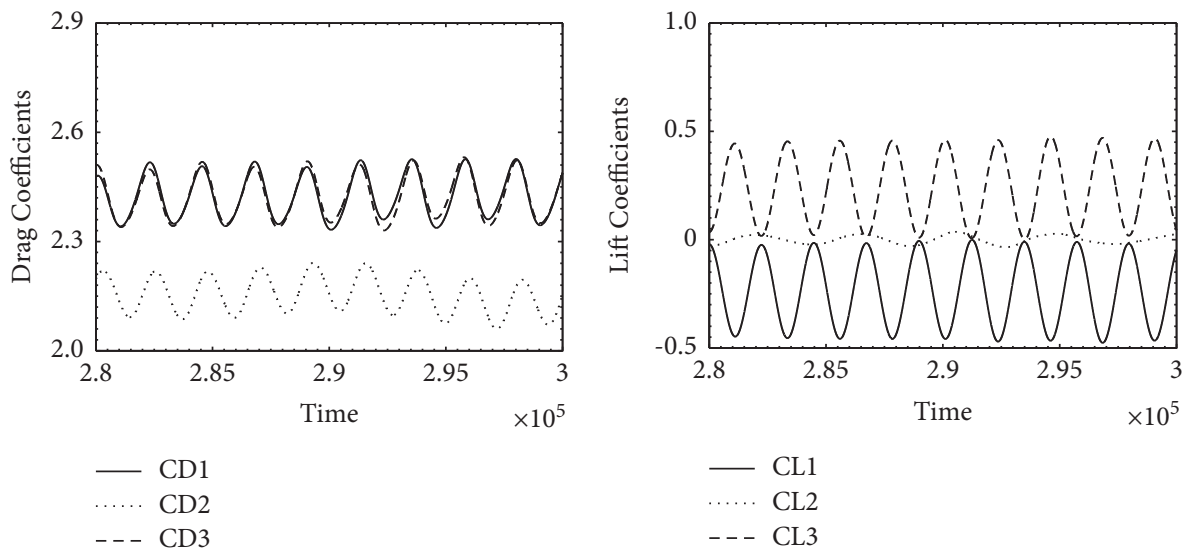

(a)

(b)
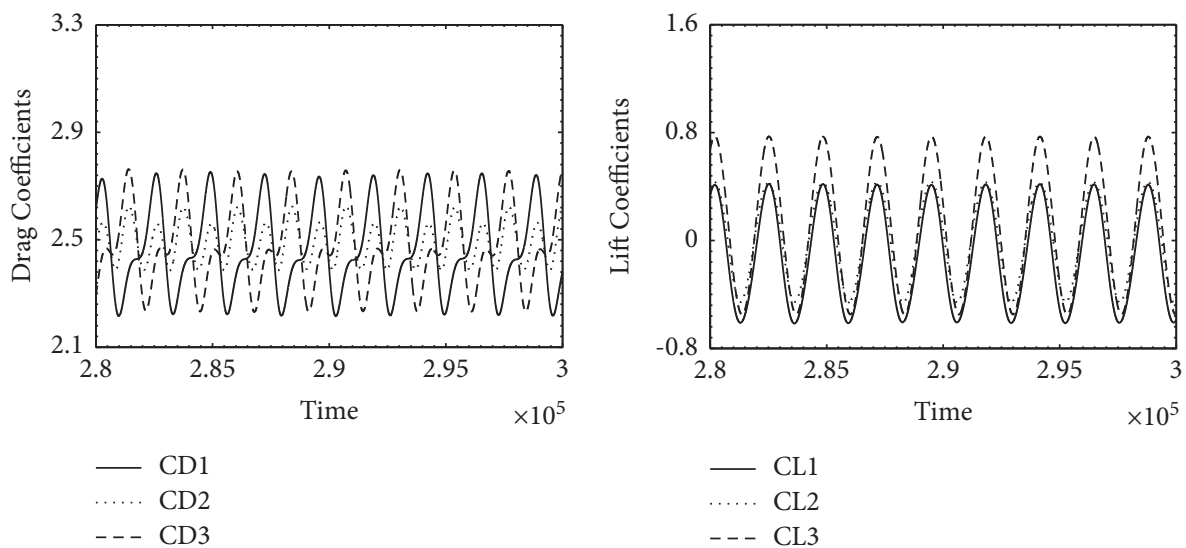

(c)

(d)
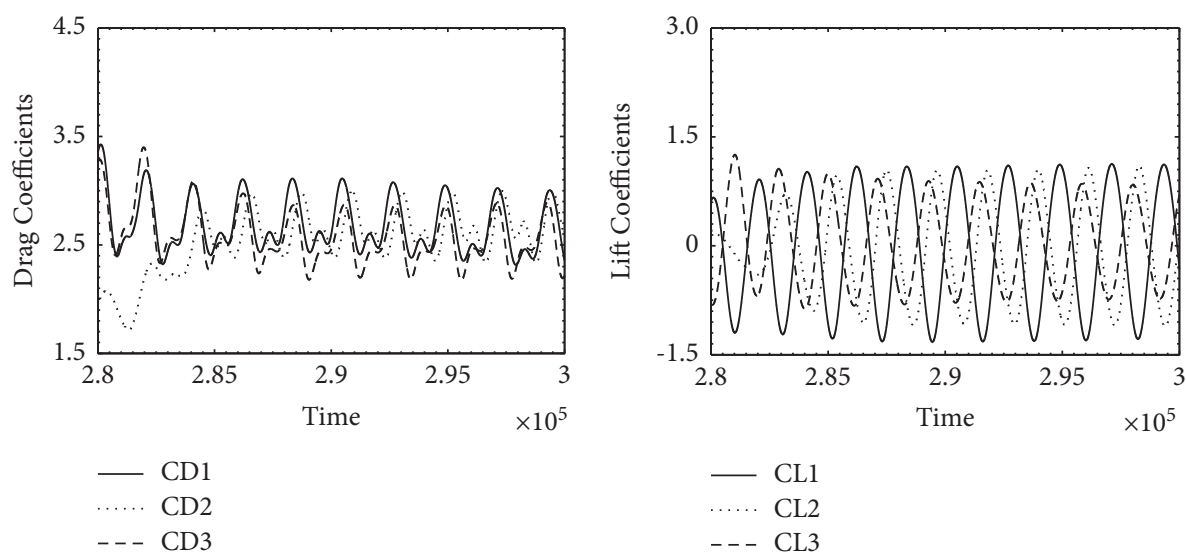

(e)

(f)

FIgURE 3: Continued. 


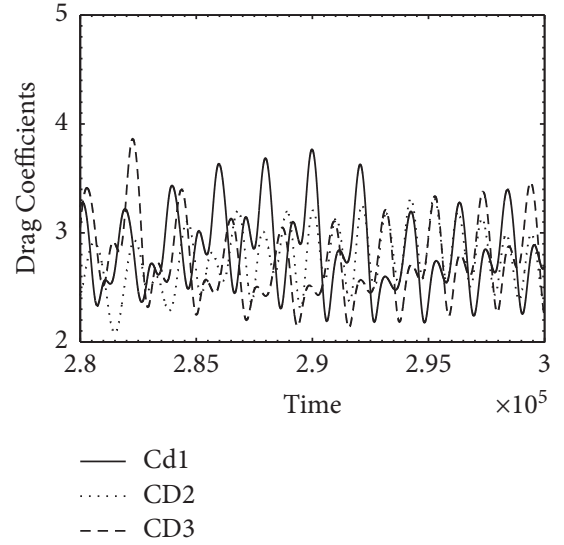

(g)

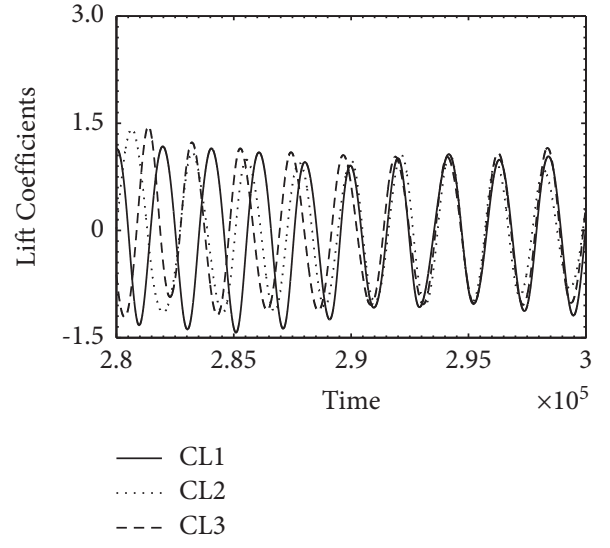

(h)

Figure 3: Signals of drag and lift coefficients for regular vortex shedding flow. (a) $(A R, R e)=(0.5,60),(b),(A R, R e)=(0.5,60),(c)(A R$, $\mathrm{Re})=(0.5,100),(\mathrm{d})(\mathrm{AR}, \mathrm{Re})=(0.5,100),(\mathrm{e})(\mathrm{AR}, \mathrm{Re})=(0.5,140),(\mathrm{f})(\mathrm{AR}, \mathrm{Re})=(0.5,140),(\mathrm{g})(\mathrm{AR}, \mathrm{Re})=(0.5,180)$, and $(\mathrm{h})(\mathrm{AR}, \mathrm{Re})=(0.5$, 180).

antiphase, and combined in-and-antiphase flow patterns indicates the regular vortex shedding from cylinders. The generated vortices show amalgamation and distortion in the wake of cylinders with different shape and size (see Figure 2). Due to this amalgamation the drag coefficient amplitude varies (see Figures 3(c), 3(e) and 3(g)). All the three cylinders' experience higher fluid forces in case of in-phase vortex shedding compared to antiphase vortex shedding (see Figures 3(c)-3(f)). The in-phase, antiphase, and combined in-and-antiphase flow patterns were also observed by [14-16] for large $g$ while we have observed the same flow pattern for intermediate $g$ due to the influence of aspect ratio.

The vorticity contours and time dependent variation of force coefficients at different values of $\operatorname{Re}$ for $A R=2$ are shown in Figures 4 and 5, respectively. Figures 4(a) and 4(b) illustrate the symmetric flow pattern in which the flow from the middle cylinder is steady. This can also be verified from the corresponding lift signals given in Figure 5(b). Figure 5(b) shows that the middle cylinders' lift coefficient is steady while those for $c_{1}$ and $c_{3}$ are antiphase to each other. The drag coefficient for this case is periodic. Also the middle cylinder experiences higher drag compared to the identical drag of lower and upper cylinders (see Figure 5(a)). For $100 \leq \mathrm{Re} \leq 180$ and $1.25 \leq \mathrm{AR} \leq 2.5$, the flow structure mechanism behind three rectangular cylinders is found to be the same and vortex shedding for these combinations is named as irregular vortex shedding. The irregular vortex shedding is further classified into modulated and flip-flopping flow patterns. Here $A R=2$ is discussed as a representative case for $100 \leq \operatorname{Re} \leq 180$. At $(A R, R e)=(2,100)$ and $(2,120)$, the vorticity contours show the irregularity of vortices in the near wake region (Figures 4(c) and 4(d)). Also the vortices from triad do not have any relationship with each other, as was observed for the case of $\mathrm{AR}=0.5$, throughout the computational domain (see Figures 4(c) and 4(d)). That is why this flow pattern is named as modulated flow pattern. From the time-trace analysis of CD and CL of modulated flow, given in Figures 5(c) and 5(d), it is clear that the variation of CD and CL is random and does not follow any pattern. This is because of the strong effect of jet flow between the gaps which destroys the shape and structure of vortices (Figures 4(c) and 4(d)). It can be seen from Figures 5(c) and 5(d) that the drag and lift signals are modulated, unsteady with varying cycles. For $(A R, R e)=(2$, $140),(2,160)$, and $(2,180)$ the flow pattern observed for irregular vortex shedding is flip-flopping in which vorticity plots show a very complex flow due to the strong effect of jet in gaps (Figures $4(\mathrm{e})-4(\mathrm{~g}))$. The generated jet from gaps bursts the vortices and no distinct vortices are observed at downstream of the computational domain (Figures 4(e)-4(g)). One important property of the flip-flopping flow is that the two gap flows asymmetrically merged and deflected to different sides and this property is clearly visible in Figures 4(e) and 4(g). Both the drag and lift are modulated and show chaotic behavior but it can be seen that the drag is more chaotic compared to lift and also the period of drag and lift coefficients is not the same (Figures 5(e) and 5(f)). The flip-flopping flow pattern was reported by Han et al. [14] and Kang [15] for flow behind three circular cylinders while Rahman et al. [16] for three square cylinders. The drag and lift signals of the flip-flopping flow pattern show irregular variations due to complex flow structure (Figures 5(e) and 5(f)). The time-trace analysis of drag shows more complex behavior compared to lift. In flip-flopping flow pattern, the distortion and merging of vortices are due to decoupling of wake dynamics of $\mathrm{CD}$ and CL.

It should be noted here that, for $60 \leq \mathrm{Re} \leq 180$ and $\mathrm{AR}=3-4$, the flow around cylinders was either symmetric or nearly symmetric similar to that discussed above. So we have not shown the vorticity and force coefficients graphs of these cases for the conciseness.

5.2. Force Statistics. This section represents the variation of fluid forces like mean drag coefficient and Strouhal number for $\mathrm{AR}=0.5,2$, and 4 and $\mathrm{Re}=60,80,100,120,140,160$, and 180. The computed results for each cylinder along with their averages are presented in Figure 6. It is important to state here that the computations are performed on the width $(w)$ 


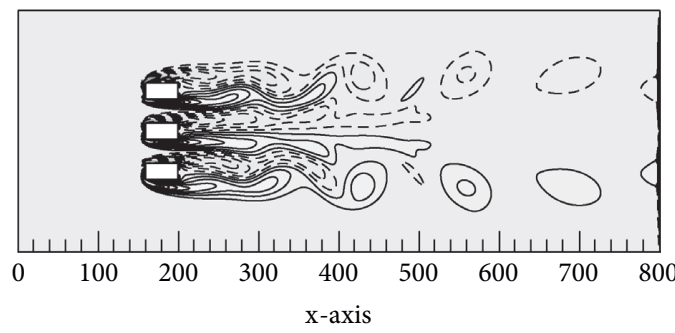

(a)

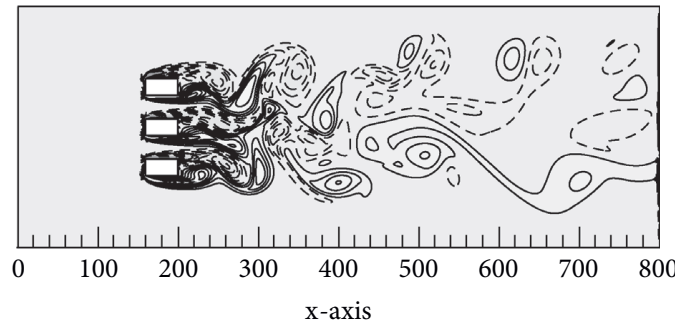

(c)

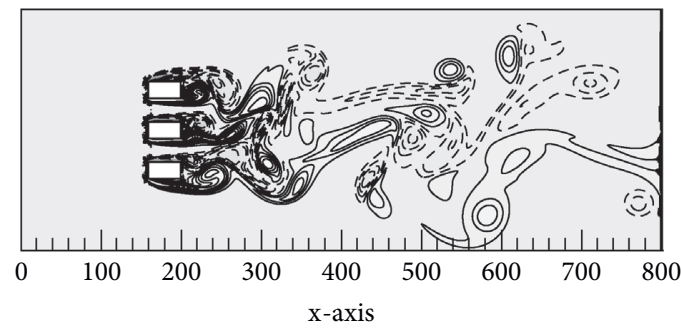

(e)

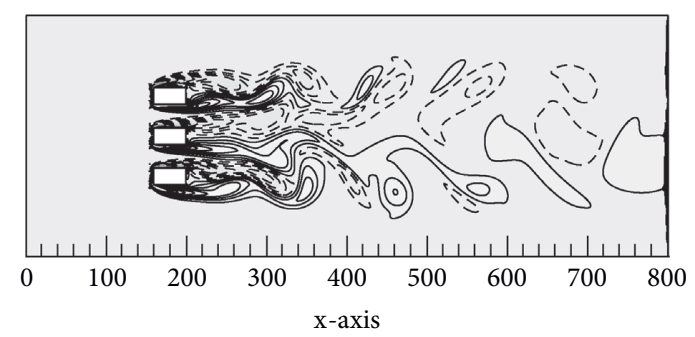

(b)

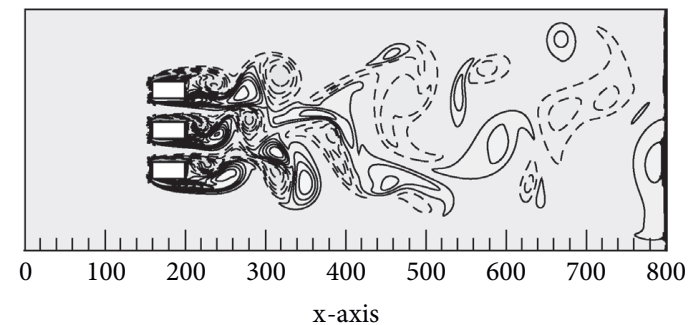

(d)

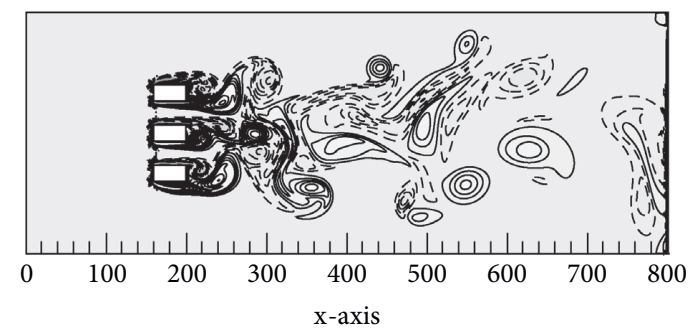

(f)

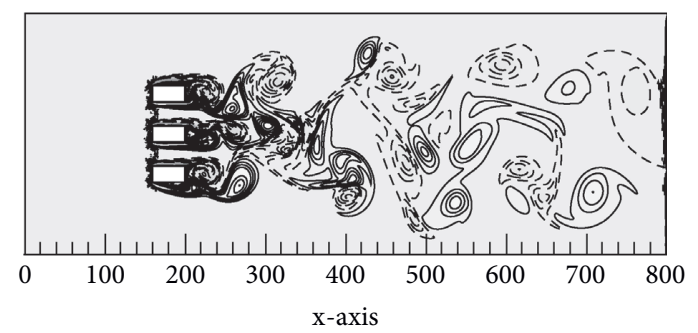

(g)

FIgURE 4: Vorticity contour visualizations at $A R=2$ and $\operatorname{Re}=60-180$. (a) $(A R, \operatorname{Re})=(2,60),(b)(A R, \operatorname{Re})=(2,80),(c)(A R, R e)=(2,100),(d)$ $(\mathrm{AR}, \mathrm{Re})=(2,120),(\mathrm{e})(\mathrm{AR}, \mathrm{Re})=(2,140),(\mathrm{f})(\mathrm{AR}, \mathrm{Re})=(2,160)$, and $(\mathrm{g})(\mathrm{AR}, \mathrm{Re})=(2,180)$.

of each cylinder in order to fully analyze the effect of AR. Comparing all three cases of AR shown here, it can be seen that the mean drag coefficient of all cylinders decreases as aspect ratio increases (Figures 6(a)-6(c)) for different Re. This indicates the fact that increment in AR stabilizes the flow and reduces the drag force acting on cylinders. Figure 6(a) shows that CDmean values of all the three cylinders increase with increment in $\operatorname{Re}$ at $\mathrm{AR}=0.5$ and CDmean1 and CDmean3 values are identical and higher from CDmean2 and CDmean(avg). It should be noted here that, at $\mathrm{AR}=0.5$ and $80 \leq \mathrm{Re} \leq 180$, the flow structure was categorized as regular vortex shedding in which complete generation of vortices was observed (see Figure 2). The increasing mean drag coefficient values with $\mathrm{Re}$, at this $\mathrm{AR}$, indicate the robustness of drag force on cylinders due to weakening viscous forces. The middle cylinder experiences higher drag as compared to upper and lower cylinders at $(\mathrm{AR}, \mathrm{Re})=2,60)$ due to jet flow effect while for other Re, this trend reverses which indicates that the increase in Re values reduces the effect of jet flow on the middle cylinder (see Figure 6(b)). The upper and lower cylinders mean drag values are identical for $\mathrm{AR}=2$ and 4 due to symmetry in flow. The middle cylinder drag for $\mathrm{AR}=0.5$ exhibits the increasing trend for increasing Re but for $\mathrm{AR}=2$ and 4, this trend reverses due to change in AR (Figures 6(a)-6(c)). It can be seen from Figure $6(\mathrm{a})$ that, at $(\mathrm{AR}, \mathrm{Re})=(0.5,60-180)$, the lower and upper cylinders experience identical drag which is higher than the drag of middle cylinders. The drag of all three cylinders is higher than their average drag (Figure 6(a)). At $\mathrm{AR}=2$, the same behavior is observed at $\mathrm{Re}=80-180$ and also the average drag curve decreases by increasing $\operatorname{Re}($ see Figure $6(\mathrm{~b}))$. At $(A R, R e)=(4,60-100)$, 


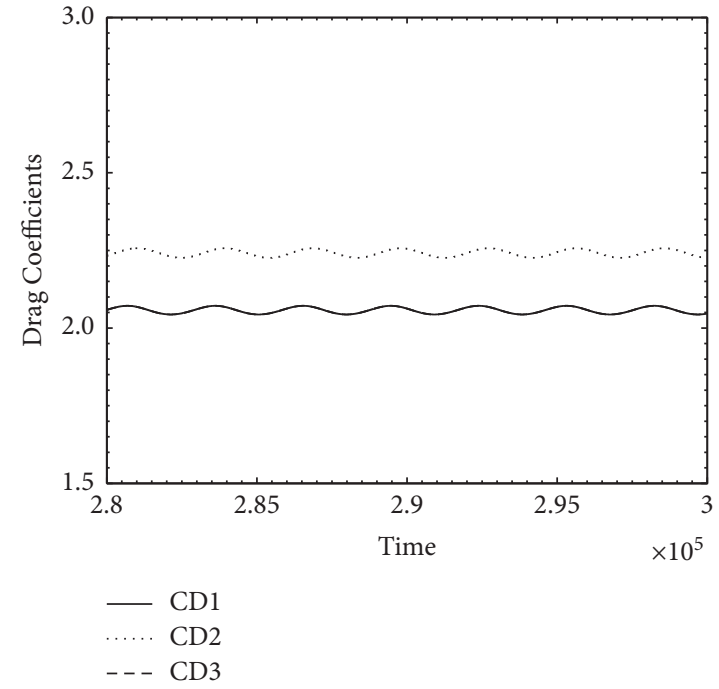

(a)

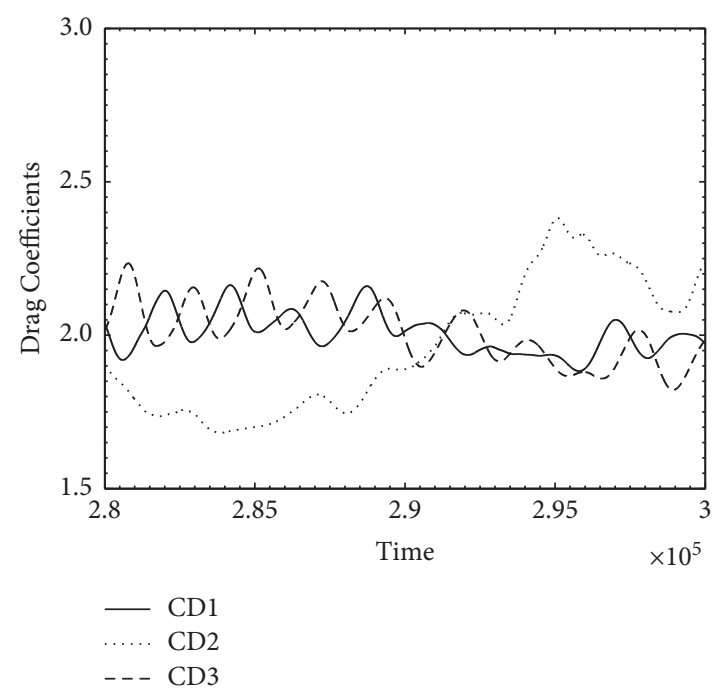

(c)

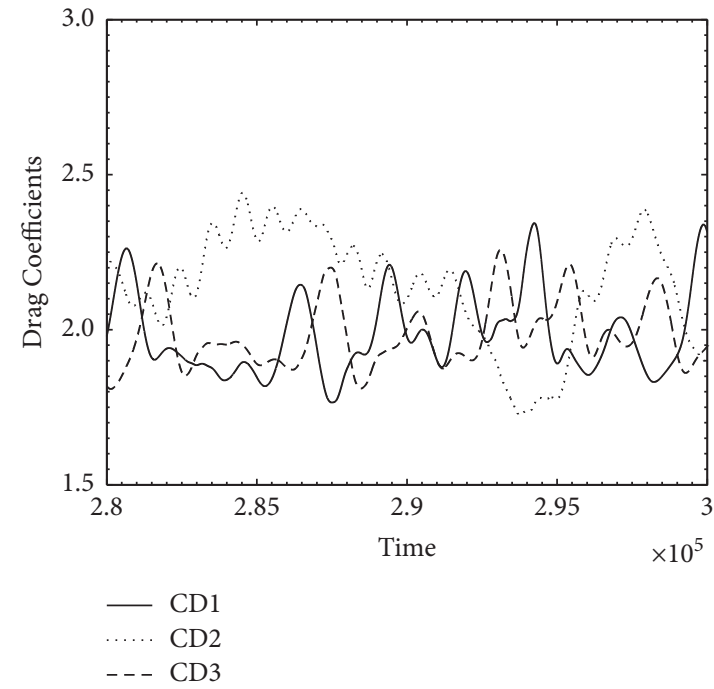

(e)

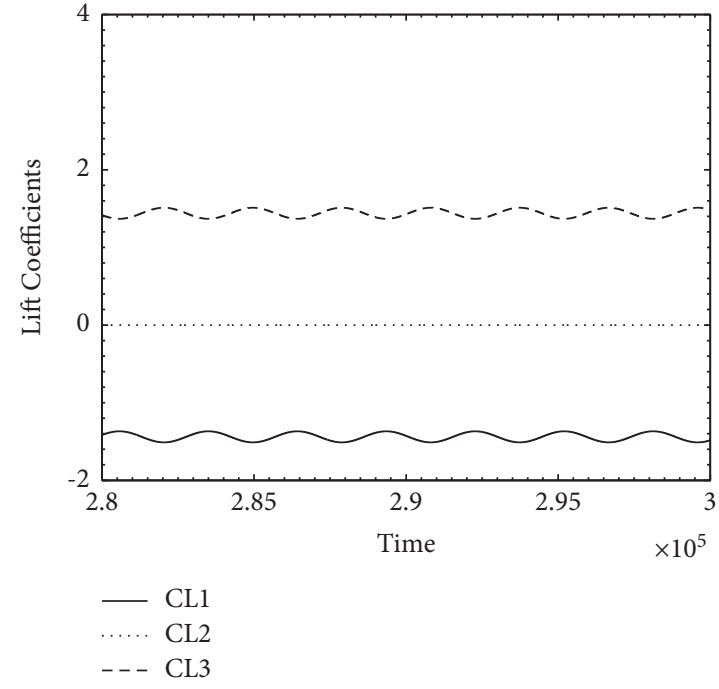

(b)

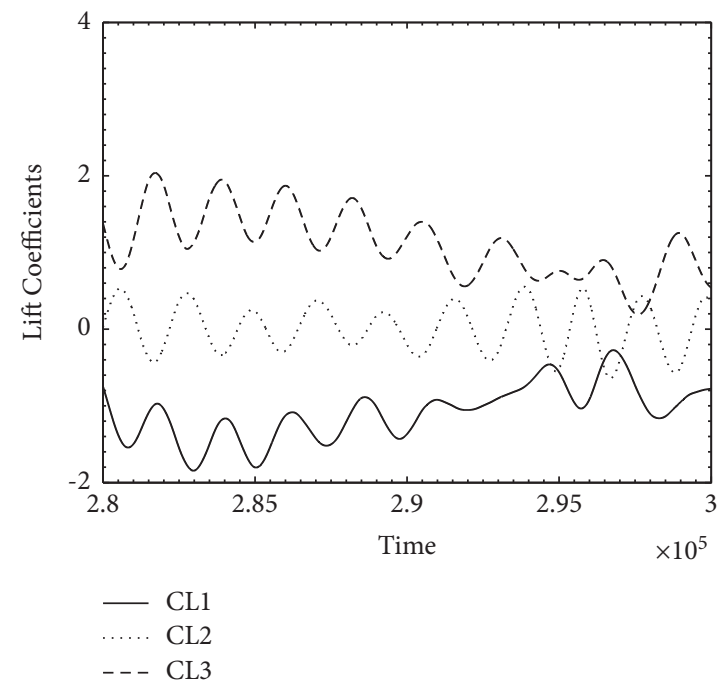

(d)

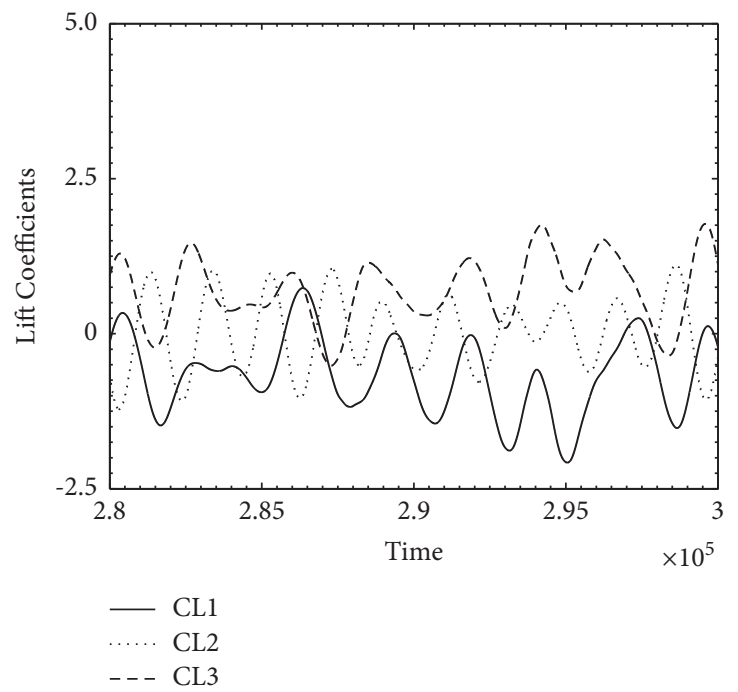

(f)

Figure 5: Temporal variations of $C D$ and CL for irregular vortex shedding flow. (a) $(A R, R e)=(2,60),(b)(A R, R e)=(2,60),(c)(A R$, $\mathrm{Re})=(2,120),(\mathrm{d})(\mathrm{AR}, \mathrm{Re})=(2,120),(\mathrm{e})(\mathrm{AR}, \mathrm{Re})=(2,160)$, and $(\mathrm{f})(\mathrm{AR}, \mathrm{Re})=(2,160)$. 




(a)

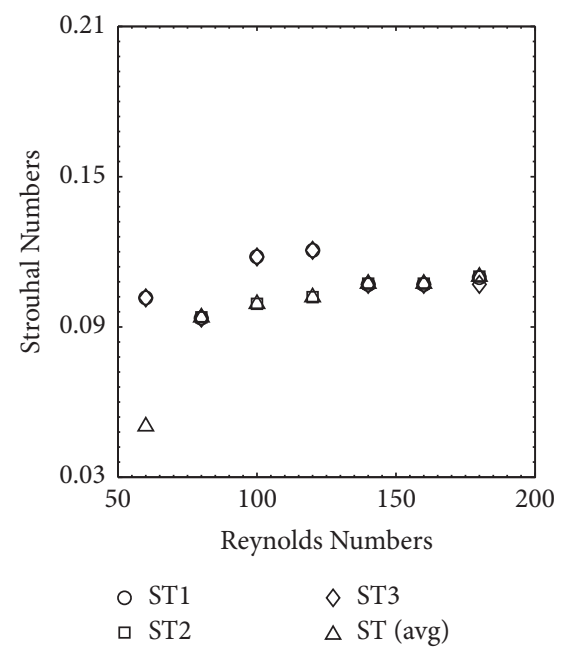

(d)



(b)

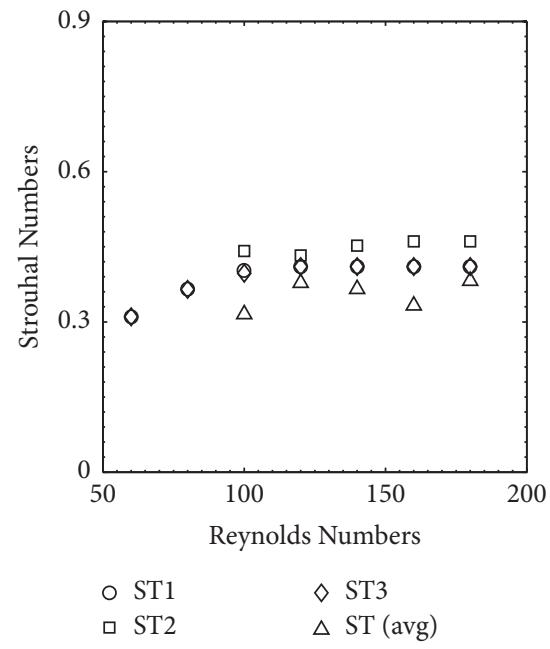

(e)

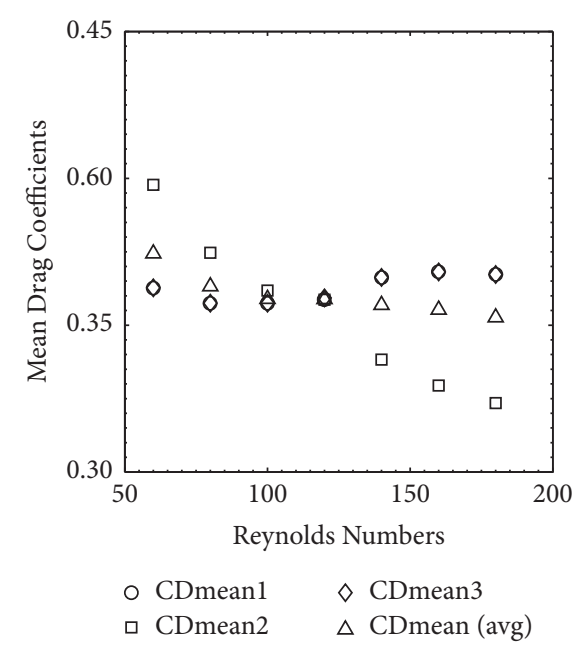

(c)

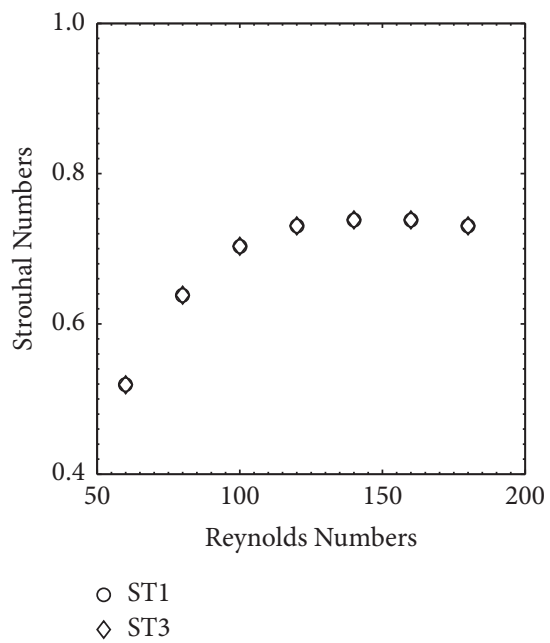

(f)

Figure 6: Effect of Re on $(a-c)$ mean drag coefficients and (d-f) Strouhal numbers, for three rectangular cylinders: $(a) A R=0.5$, (b) $A R=2$, (c) $\mathrm{AR}=4$, (d) $\mathrm{AR}=0.5$, (e) $\mathrm{AR}=2$, and (f) $\mathrm{AR}=4$.

the average drag is higher than the drags of all three cylinders, at $(\mathrm{AR}, \mathrm{Re})=(4,120)$, all the cylinders drag and the average drag coincide, and at $(\mathrm{AR}, \mathrm{Re})=(4,140-180)$, the average drag is higher than the drags of all three cylinders (see Figure 6(c)). The local maximum CDmean at all values of AR and Re can be seen for $c_{1}$ and $c_{3}$ to be 5.9075 at (AR, $\mathrm{Re})=(0.5,180)$. At this combination of AR and Re, the flow pattern was combined in-and-antiphase vortex shedding. The local minimum value can be seen at $(\mathrm{AR}, \mathrm{Re})=(4,180)$ for cylinder $c_{2}$, where symmetric flow behavior was observed. From Figure 6(c) it can also be seen that, at (AR, $\mathrm{Re})=(4,120)$, the CDmean of all cylinders coincides. Reason for this similarity is that, due to larger AR, symmetry develops in flow around each cylinder which results in similarity of drag acting on cylinders. Also, it should be noted that at this combination of AR and Re nearly symmetric flow pattern was observed. This observation indicates the role of AR in stability of fluid forces.

Variation of ST for all cylinders with Re at different values of $A R$ is shown in Figures 6(d)-6(f). For nearly symmetric flow pattern the ST values of upper and lower cylinders are identical and the middle cylinder ST values are not calculated due to steady flow (see Figure 6(f)). Comparison of Figures 6(d)-6(f) reveals that the ST for all cylinders increases with increment in AR. This indicates that as AR increases the vortices shed from the cylinders at higher frequency. The local maximum value of ST for the chosen cases is 0.7385 , observed for both $c_{1}$ and $c_{3}$ at $(\mathrm{AR}, \mathrm{Re})=(4$, 140). The flow pattern for this case was nearly symmetric while the local minimum value 0.0938 for the ST is observed at $(\mathrm{AR}, \mathrm{Re})=(0.5,80)$, for middle cylinder, where the flow pattern was combined in-and-antiphase vortex shedding. In this flow pattern the formation of vortices behind the middle cylinder was weaker compared to the upper and lower cylinders due to small Re. The average ST is identical to the middle cylinder ST and smaller than ST1 and ST2 for (AR, $\mathrm{Re})=(0.5,100-120)$, while for $(\mathrm{AR}, \mathrm{Re})=(0.5,140-180)$, all the cylinders ST and their average are the same (see Figure $6(\mathrm{~d}))$. At $(\mathrm{AR}, \mathrm{Re})=(2,100-180)$, the middle cylinder ST is higher than ST of lower and upper cylinders and all 
three cylinders ST are higher than their average ST (see Figure 6(e)). Figure 6 also shows that for all cases of AR the ST monotonically increases with increment in Re. This is due to the fact that the dominancy of inertial forces results in maximizing the vortex shedding frequency.

\section{Conclusions}

In this paper, the numerical simulations for flow around three rectangular cylinders arranged side-by-side were presented in order to fully analyze the effect of Reynolds number on flow structures and fluid forces at different aspect ratios and fixed spacing ratio of 1.5 using LBM. The Reynolds number was varied in the range $60 \leq \operatorname{Re} \leq 180$ and twelve different values of AR between 0.25 and 4 were selected. Different flow patterns, variation of fluid forces, and vortex shedding frequency were documented and analyzed in terms of the jet effects between the gaps, downstream flow characteristics, and alteration of the wake structures at different Re and AR in this work. The vortex shedding mechanism behind cylinders was categorized into regular $\quad(0.25 \leq \mathrm{AR} \leq 1 ; \quad 80 \leq \mathrm{Re} \leq 180), \quad$ irregular $(1.25 \leq \mathrm{AR} \leq 2.5 ; 100 \leq \mathrm{Re} \leq 180)$, and symmetric vortex shedding $(3 \leq \mathrm{AR} \leq 4 ; 60 \leq \mathrm{Re} \leq 180, \mathrm{Re}=60 ; 1.25 \leq \mathrm{AR} \leq 2.5$ and $\operatorname{Re}=60,80 ; 0.25 \leq A R \leq 1)$. The regular vortex shedding was further subdivided into in-phase, antiphase, and combined inand-antiphase vortex flow patterns. The irregular vortex shedding was further subdivided into modulated and flipflopping flow patterns while symmetric vortex shedding was categorized into symmetric and nearly symmetric flow patterns. In regular and symmetric vortex shedding patterns the drag and lift coefficients were found to vary smoothly while in irregular vortex shedding the behavior of force coefficients was abruptly changing with time. It was found that the mean drag coefficients of the three rectangular cylinders decreased while shedding frequencies increased for increasing AR at all Re. The upper and lower cylinders experienced identical drag for symmetric vortex shedding. Moreover, at $\mathrm{AR}=0.5$, all the cylinders had a higher magnitude of drag compared to $A R=2$ and 4 . In symmetric vortex shedding the lift signals for the middle cylinder became constant due to steady flow behavior. Also, it was observed that the ST values monotonically increase with increment in Re for all cases of AR. AR was found to have significant effects on flow characteristics.

\section{Data Availability}

The data that support the findings of this study are available from the corresponding author, Zeeshan Alam, upon reasonable request.

\section{Conflicts of Interest}

The authors declare that they have no conflicts of interest.

\section{References}

[1] S. U. Islam, W. S. Abbasi, and H. Rahman, "Force statistics and wake structure mechanism of flow around a square cylinder at low Reynolds numbers," International Journal of
Mechanical, Aerospace, Industrial and Mechatronics Engineering, vol. 8, no. 8, pp. 1417-1423, 2014.

[2] R. Golani and A. Dhiman, "Fluid flow and heat transfer across a circular cylinder in the unsteady flow regime," International Journal of Engineering Science, vol. 3, no. 3, pp. 8-19, 2004.

[3] B. Gera, P. K. Sharma, and R. K. Singh, "CFD analysis of 2D unsteady flow around a square cylinder," International Journal of Applied Engineering Research, vol. 1, no. 3, p. 602, 2010.

[4] S. U. Islam, C. Y. Zhou, A. Shah, and P. Xie, "Numerical simulation of flow past rectangular cylinders with different aspect ratios using the incompressible lattice Boltzmann method," Journal of Mechanical Science and Technology, vol. 26, no. 4, pp. 1027-1041, 2012.

[5] S. Abdollah, A. F. Mahdi, and R. Noorallah, "Experimental study of near wake flow behind a rectangular cylinder," American Journal of Applied Sciences, vol. 5, no. 8, pp. 917926, 2008.

[6] A. Okajima, "Numerical simulation of flow around rectangular cylinders," Journal of Wind Engineering and Industrial Aerodynamics, vol. 33, no. 1-2, pp. 171-180, 1990.

[7] A. Agrawal, L. Djenidi, and R. A. Antonia, "Investigation of flow around a pair of side-by-side square cylinders using the lattice Boltzmann method," Computers \& Fluids, vol. 35, no. 10, pp. 1093-1107, 2006.

[8] S. Kang, "Characteristics of flow over two circular cylinders in a side-by-side arrangement at low Reynolds numbers," Physics of Fluids, vol. 15, no. 9, pp. 2486-2498, 2003.

[9] M. M. Alam and Y. Zhou, "Intrinsic features of flow around two side-by-side square cylinders," Physics of Fluids, vol. 25, no. 8, Article ID 085106, 2013.

[10] Z. Han, D. Zhou, J. Tu, C. Fang, and T. He, "Flow over two side-by-side square cylinders by CBS finite element scheme of Spalart-Allmaras model," Ocean Engineering, vol. 87, pp. 40-49, 2014.

[11] L. Zhang, X. Mao, and L. Ding, "Influence of attack angle on vortex-induced vibration and energy harvesting of two cylinders in side-by-side arrangement," Advances in Mechanical Engineering, vol. 11, no. 1, 2019.

[12] C. M. Sewatkar, A. Sharma, and A. Agrawal, "On the effect of Reynolds number for flow around a row of square cylinders," Physics of Fluids, vol. 21, no. 8, Article ID 083602, 2009.

[13] A. B. Harichandan and A. Roy, "Numerical investigation of low Reynolds number flow past two and three circular cylinders using unstructured grid CFR scheme," International Journal of Heat and Fluid Flow, vol. 31, no. 2, pp. 154-171, 2010.

[14] Z. Han, D. Zhou, and J. Tu, "Laminar flow patterns around three side-by-side arranged circular cylinders using semiimplicit three-step Taylor-characteristic-based-split (3-TCBS) algorithm," Engineering Applications of Computational Fluid Mechanics, vol. 7, no. 1, pp. 1-12, 2013.

[15] S. Kang, "Numerical study on laminar flow over three side-byside cylinders," KSME International Journal, vol. 18, no. 10, pp. 1869-1879, 2004.

[16] H. Rahman, S. U. Shams-Ul-Islam, C. Y. Zhou, T. Kiyani, and S. C. Saha, "On the effect of Reynolds number for flow past three side-by-side square cylinders for unequal gap spacings," KSCE Journal of Civil Engineering, vol. 19, no. 1, pp. 233-247, 2015.

[17] S. Ul Islam, H. Rahman, Z. C. Ying, and S. C. Saha, "Comparison of wake structures and force measurements behind three side-by-side cylinders," Journal of the Brazilian Society 
of Mechanical Sciences and Engineering, vol. 38, no. 3, pp. 843-858, 2016.

[18] H. Rahman, S. U. Islam, W. S. Abbasi, S. Mukhtar, and T. Alam, "Flow structure mechanism around three rectangular bodies using the lattice Boltzmann method," Ocean Engineering, vol. 216, no. 1, pp. 1-11, Article ID 108101, 2020.

[19] H. Rahman, W. S. Abbasi, S. U. Islam, R. Khan, and M. U. Khan, "Flow features of three side-by-side rectangular cylinders under the effect of aspect ratios and Reynolds numbers," International Journal of Modern Physics C, vol. 32, no. 3, pp. 1-19, Article ID 21550034, 2021.

[20] M. C. Sukop and D. T. Thorne, Lattice Boltzmann Modeling: An Introduction for Geoscientists and Engineers, Springer, Berlin, Germany, 2007.

[21] D. A. Wolf-Gladrow, Lattice-Gas Cellular Automata and Lattice Boltzmann Models: An Introduction, Springer, Berlin, Germany, 2004.

[22] A. A. Mohamad, Lattice Boltzmann Method: Fundamentals and Engineering Applications with Computer Codes, Springer, Berlin, Germany, 2011.

[23] Z. Guo, B. Shi, and N. Wang, "Lattice BGK model for incompressible Navier-Stokes equation," Journal of Computational Physics, vol. 165, no. 1, pp. 288-306, 2000.

[24] S. Chapman and T. G. Cowling, The Mathematical Theory of Non-Uniform Gases, Cambridge University Press, Cambridge, UK, Third edition, 1970.

[25] M. A. Gallivan, D. R. Noble, J. G. Georgiadis, and R. O. Buckius, "An evaluation of the bounce-back boundary condition for lattice Boltzmann simulations," International Journal for Numerical Methods in Fluids, vol. 25, no. 3, pp. 249-263, 1997.

[26] A. Sohankar, C. Norberg, and L. Davidson, "Low-Reynoldsnumber flow around a square cylinder at incidence: study of blockage, onset of vortex shedding and outlet boundary condition," International Journal for Numerical Methods in Fluids, vol. 26, no. 1, pp. 39-56, 1998.

[27] A. Okajima, "Strouhal numbers of rectangular cylinders," Journal of Fluid Mechanics, vol. 123, pp. 379-398, 1982.

[28] C. Norberg, "Flow around rectangular cylinders: pressure forces and wake frequencies," Journal of Wind Engineering and Industrial Aerodynamics, vol. 49, no. 1-3, pp. 187-196, 1993.

[29] A. Sohankar, C. Norberg, and L. Davidson, "Numerical simulation of unsteady flows around a square two dimensional cylinder," in Proceedings of the Twelfth Australasian Fluid Mechanics Conference, pp. 517-520, Sydney, Australia, 1995.

[30] O. Inoue and Y. Suzuki, "Beat of sound generated by flow past three side-by-side square cylinders," Physics of Fluids, vol. 19, no. 4, Article ID 048102, 2007. 